\title{
Caracterization of Thymic Epithelial Cells During the Development of Autoimmune Encephalomyelits
}

Experimental

\section{ALEXANDRE B. PEREIRA*; JESSICA FUNARI; ALESSANDRO DOS SANTOS FARIAS; CAROLINA FRANCELIN}

\begin{abstract}
Thymus is a primary lymphoid organ responsible for T cell development and despite this unique function, it is a target organ in many different diseases. Thymus microenvironment is composed by lymphocytes and stromal components. In the stromal compartment we can find epithelial cells which are divided in two subtypes: cortical and medullar thymic epithelial cell. Such cells are important to thymocyte maturation and education as they provide the essential signals to $T$ cell development. Previously, we have reported that during the clinical course of EAE, which is a model of a T CD4+ lymphocyte-mediated autoimmunity disease, thymus present important alterations in lymphocyte compartment and neuronal molecules. In this project, we pretended caracterize the alterations in thymus stromal compartment in order to a better undestanding of thymus during an autoimmune disease.
\end{abstract}

Key words:

Immune system, epithelial cells, EAE.

\section{Introduction}

Experimental autoimune encephalomyelitis (EAE) is an autoimmune disease mediated by $\mathrm{CD}^{+} \mathrm{T}$ lymphocytes and is used as an experimental model of multiple sclerosis (MS). $\mathrm{T}$ lymphocytes are educated during its migration through thymus microenvironment, but sometimes some of then escape from central tolerance process and go to periphery able to start an autoagressive response. Thymus have a

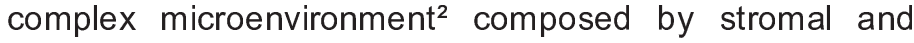
lymphocyte compartment. In the stromal compartment is possible to observe neuronal structure, suggesting that any central nervous system alteration may evoke a thymic disrupment. Since 2014, our group study thymus alteration during EAE clinic evolution; previously we reported that thymus from mice (after 15 days post immunization) presented reduction in thymus relative weight, alteration in cortical-medullary delimitation and loss of double positive thymocytes. Here in, through specific stain, we show that thymus from EAE mice present alteration in cortical and medullar thymic epithelial cell localization and in mesenchymal cell deposition. Our results add new information about thymus atrophy that might contribute to a better understanding of development of autoreactive cells in $E A E$ as well as to further knowledge about the thymic interaction at different pathological processes.

\section{Results and Discussion}

After the immunization and evolution of EAE, we sacrified our mices and prepared its thymus to (1) histological sections in order to analyze epithelial and mesenchymal cells through specific immunostaining against cytokeratins, lipid droplets and fibroblast, or (2) flow cytometry analyses in order to quantify thymic epithelial cells subtypes.

Our studies revealed that thymus from EAE mice present a major density to mesenchymal positive cells when compared to control samples and disruption of cortical and medullar areas. In flow cytometry, we used UEA-1 to identify general epithelial cells, and Ly51 for cortical epithelial cells. Our analysis revealed that in control animals, we have less than $20 \%$ of cTEC cells in thymus, while it would grown following the development of clinical sings of EAE. Also, there was a decrease of the relative number of $\mathrm{mTEC}$ when compared the control mice. Together these results suggests that during $E A E$ thymic microenvironment suffers a remodeling, altering stromal compartment ${ }^{3}$.

\section{Conclusions}

Until that moment, our data has shown that the animals that developed the EAE disease has presented atrophic thymus, followed by deep compromising in both stromal and lymphocyte compartments, that may contribute to exacerbate autoimmune reactions or to downregulate the autoagressive response.

\section{Acknowledgement}

We thank all the Neuroimmunology Unit, the Institute of Biology at Unicamp, INFABIC, Wilson Savino's group, FAPESP and CNPQ.
1.Sospedra, M. \& Martin, R. Annu. Rev. Immunol. 23, 683-747 (2005).

2.Manley, N. R., Richie, E. R., Blackburn, C. C., Condie, B. G. \& Sage, J. Front Biosci (Landmark Ed) 16, 2461-2477 (2011).

3.Inami. Y; et al. Immunology and Cell Biology (2011) 89, 314-321; (2010) 